\title{
Performance Evaluation of Unified Power Flow Controller using Transient Simulation
}

\author{
A.M.Kulkarni $\quad$ K.R.Padiyar \\ Department of Electrical Engineering \\ Indian Institute of Science \\ Bangalore, India 560012
}

\begin{abstract}
The Unified Power Flow Controller (UPFC) is a power electronic controller which can be used to control active and reactive power flows in a transmission line by injection of (variable) voltage in series and reactive current in shunt. In this paper we evaluate the performance of a control strategy for UPFC in which we control real power flow through the line, while regulating magnitudes of the voltages at its two ports using transient simulation.

The real power reference is modulated in order to improve the damping of power swings in the line. The effectiveness of the UPFC with this control strategy is clearly brought out by the simulation study

Topic:Applications: FACTS; Computer Simulation.
\end{abstract}

\section{Introduction}

The rapid development of power electronics has made it possible to use high power electronic equipment for high voltage systems. The concept of Flexible AC Transmission Systems (FACTS) envisages the use of solid state controllers to achieve flexibility of power system operation by fast and reliable control. The Unified Power Flow Controller (UPFC) $[1,2]$ is the most versatile of the FACTS controllers envisaged so far. The main function of the UPFC is to control the flow of real and reactive power by injection of a voltage in series with the transmission line. Both the magnitude and the phase angle of the voltage can be varied independently. In addition UPFC can also inject controllable reactive current to regulate the voltage at one port of UPFC. Real and reactive power flow control can allow for regulation of power flow in prescribed routes, loading of transmission lines closer to their thermal limits and can be utilized for improving transient and small signal stability of the power system.

The schematic of the UPFC is shown in Fig.1. The UPFC consists of 2 branches. The series branch consists of a voltage source converter which injects a voltage in series through a transformer. Since the series branch of the UPFC can inject a voltage with variable magnitude and phase angle it can exchange real power with the transmission line. However the UPFC as a whole cannot supply or absorb real power in steady state (except for the power drawn to com-

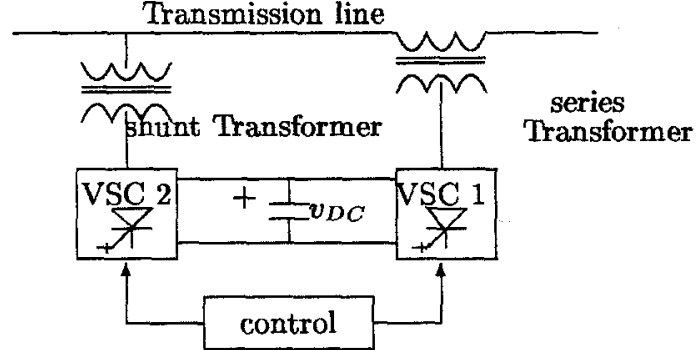

Figure 1: UPFC

pensate for the losses) unless it has a power source at its DC terminals. Thus the shunt branch is required to compensate (from the system) for any real power drawn/ supplied by the series branch and the losses. If the power balance is not maintained, the capacitor cannot remain at a constant voltage. The relationship can be expressed mathematically as (see Fig. 2):

$$
\Re\left(\overline{V^{u 1}} \overline{I_{1}^{*}}+\overline{V^{u 2}} \overline{I_{2}^{*}}\right)-P_{\text {loss }}=0
$$

where $P_{\text {loss }}$ is the cumulative sum of losses in the UPFC including those in the converter, capacitor and transformers.

In addition to maintaining the real power balance, the

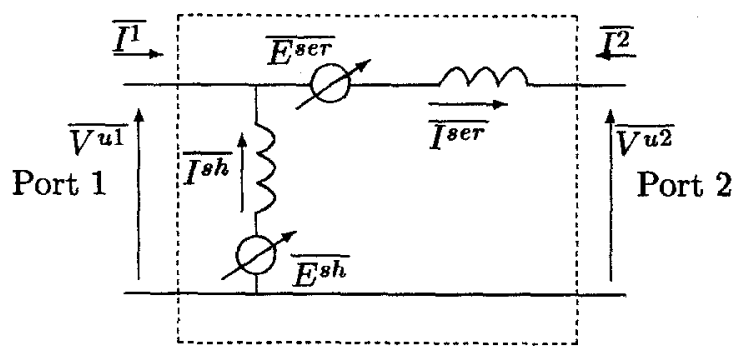

Figure 2: UPFC as a two port device

shunt branch can independently exchange reactive power with the system. A control strategy for UPFC has been described in $[3,4]$ in which the real power flow in the line is 
controlled while regulating the magnitudes of the voltages at the two ports of the UPFC. The controllers use only local measurements. In this paper we evaluate the performance of UPFC employing this control scheme using transient digital simulation for a case study.

\section{A Control Scheme for UPFC}

The main advantage of the power electronics based FACTS controllers over mechanical controllers is their speed. Therefore the capabilities of the UPFC need to be exploited not only for steady state load flow control but also to improve stability. However it is not obvious as to how to use the voltage and current (subject to the power balance constraint) for UPFC control. The UPFC allows us three "degrees of freedom"

1. Magnitude and angle of series voltage

\section{Shunt reactive current.}

The UPFC uses Voltage Source Converters (VSCs) for series voltage injection as well as shunt current control. The injection of series voltage can respond almost instantaneously to an order. The shunt current is controlled indirectly by varying the shunt converter voltage (closed loop control of shunt current is required). Thus the series voltage can be considered as an input directly while the shunt current controller has to be designed.

The real and reactive power flow in the line can be controlled independently using the series injected voltage [1]. The real and power flow in a transmission line is given in terms of (magnitude of) voltage at the two ends of the transmission line and the angle between them (see Fig. 3):

$$
\begin{gathered}
P_{1}=-P_{2}=\frac{V_{1} V_{2} \sin \left(\phi_{1}-\phi_{2}\right)}{X} \\
Q_{1}=\frac{V_{1}^{2}}{X}-\frac{V_{1} V_{2} \cos \left(\phi_{1}-\phi_{2}\right)}{X} \\
Q_{2}=\frac{V_{2}^{2}}{X}-\frac{V_{1} V_{2} \cos \left(\phi_{1}-\phi_{2}\right)}{X}
\end{gathered}
$$

UPFC can control all the three basic quantities (voltage magnitude, transmission line angle and reactance). It can be thought of as a combination of STATic CONdenser (STATCON) which is a shunt reactive compensation device and Series Synchronous Static Compensator(SSSC) which is a series reactance compensation device, with an additional degree of freedom of real voltage injection. In fact UPFC can perform all the functions of STATCON , SSSC and TCPAR (Thyristor Controlled Phase angle regulator).

\subsection{Series injected voltage control}

To achieve real and reactive power flow control we need to inject series voltage of the appropriate magnitude and angle. The injected voltage can be split into two components which are in phase ("real voltage: $e_{P}^{g e r ")}$ ) and in quadrature ("reactive voltage $: e_{R}^{s e r}$ ") with the line current.

Injected reactive and real voltages are written in terms of

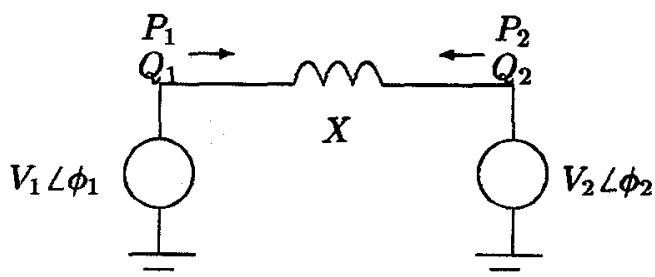

Figure 3: Transmission line

injected voltages in the D-Q (synchronously rotating at system frequency) frame $\left(e_{D}^{s e r}, e_{Q}^{s e r}\right)$ as,

$$
\begin{aligned}
& e_{R}^{s e r}=e_{D}^{s e r} \cos \left(\phi^{i}\right)-e_{Q}^{s e r} \sin \left(\phi^{i}\right) \\
& e_{P}^{s e r}=e_{D}^{s e r} \sin \left(\phi^{i}\right)+e_{Q}^{g e r} \cos \left(\phi^{i}\right)
\end{aligned}
$$

where $\phi^{i}=\tan ^{-1} \frac{i_{D}^{\text {ser }}}{i_{Q}^{\text {ser }}}$ is the angle of the current in the D-Q frame.

It is to be noted that the line current measurement is locally available. The real power can be effectively controlled by varying the series reactance of the line (Eq. 2). Reactive voltage injection is equivalent to insertion of reactance in the line; however the voltage injected can be independent of the transmission line current. Thus real power is controlled using the reactive voltage and the reactive power is controlled using the real voltage.

It is necessary to distinguish between the role of the UPFC as a power flow controller in order to achieve steady state objectives (slow control) and as a device to improve transient performance (requiring fast control.) Thus, while real and reactive power references are set from the (steady state) load flow requirements, the real power reference can also be modulated to improve damping and transient stability. In addition, reactive power can be controlled to prevent dynamic over/undervoltages. In fact, instead of having closed loop control of reactive power using the real voltage, the voltage at port 2 of the UPFC can be controlled readily by calculating the required real voltage to be injected. We can control reactive power indirectly by changing the voltage reference for port2. The structure of the real power controller is shown in Fig. 4. An auxiliary current angle signal $\left(\phi^{i}\right)$ is used to improve the stability of the mode associated with the series inductance of the line. This has no contribution in steady state.

A detailed description of the controller design is given in

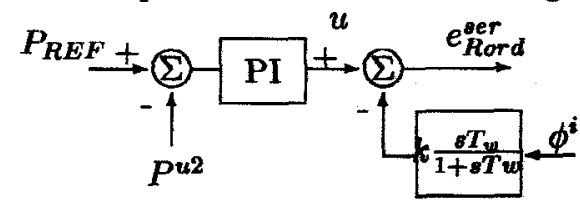

Figure 4: Real Power Controller

[3].

\subsection{Shunt Current Control}

It is well known that shunt reactive power injection can be used to control bus voltage. Thus the shunt current is 


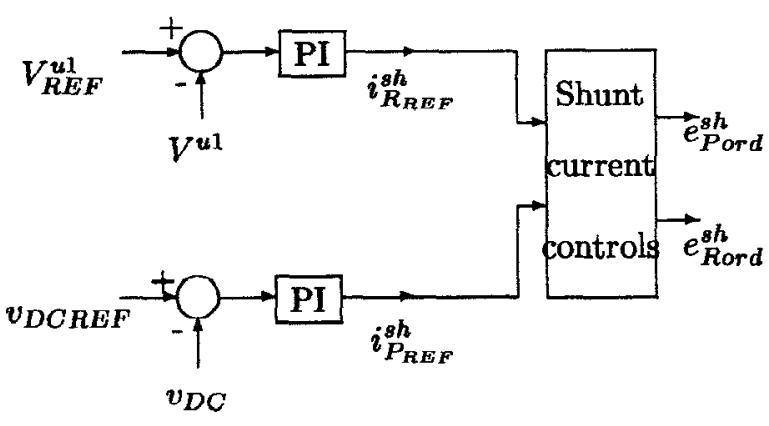

Figure 5: shunt current controller

split into real (in phase with bus voltage $: i_{P}^{s h}$ ) and reactive current $\left(i_{R}^{s h}\right)$ components. Reactive and real currents are written in terms of currents drawn from port 1 of UPFC in the D-Q frame $\left(i_{D}^{s h}, i_{Q}^{s h}\right)$ as,

$$
\begin{aligned}
& i_{R}^{s h}=i_{D}^{s h} \cos \left(\theta^{u 1}\right)-i_{Q}^{\text {gh }} \sin \left(\theta^{u 1}\right) \\
& i_{P}^{s h}=i_{D}^{s h} \sin \left(\theta^{u 1}\right)+i_{Q}^{\text {sh }} \cos \left(\theta^{u 1}\right)
\end{aligned}
$$

where $\theta^{u 1}=\tan ^{-1} \frac{v_{D}^{u 1}}{v_{Q}^{u 1}}$ is the angle of the voltage at port 1 of the UPFC in the D-Q frame.

The reference value for the real current is set so that the capacitor voltage is regulated (which implies power balance). The reactive current reference is set by a bus voltage magnitude regulator (for port 1 of the UPFC). The voltage reference of the voltage regulator itself can be varied (slowly) so as to meet steady state reactive power requirements.

For control of shunt current we proceed in a way similar to the one outlined by Schauder and Mehta[5]. The controller block diagram is shown in Fig. 5.

\subsection{Modulation Controller[4]}

Following a disturbance in the power system, the generator rotor tends to oscillate and this oscillation is often poorly damped. Moreover a large disturbance may even cause loss of synchronism. In order to improve stability, UPFC controls the power flow so as to set up restoring torques which oppose the motion. This is done by modulating the power reference of the UPFC in phase with the speed deviation of the generator (damping action $D$ ) in addition to a component in phase with the generator angle deviation (synchronising action $K$ ). The block diagram of the modulation controller is shown in Fig. 6. The modulation signal is the generator rotor slip. A washout circuit is provided so as to prevent any steady state bias.

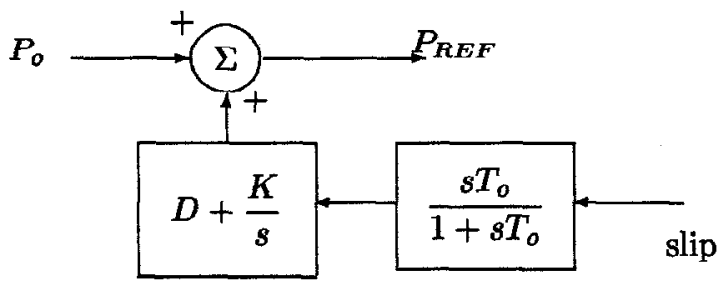

Figure 6: Modulation Controller

\section{Case Study}

\subsection{Converter Configuration}

The voltage source converters are required to provide variable magnitude and angle of voltage. The converters can be broadly classified into multilevel and multipulse configuration. Magnitude control can be achieved by some form of PWM. We have considered, for the purpose of evaluation of the control strategy, magnitude control by addition of the output of 2 twelve pulse converters which are mutually phase displaced by some angle, while maintaining the required phase angle $(\phi)$ of the resultant voltage [6]. The phasor diagram is shown in Fig. 7 .

The magnitude of the converter ac voltage is related to the DC voltage by a factor $\frac{2 \sqrt{6}}{\pi}$ for a 12 pulse converter. Thus the resultant voltage $\bar{E}$ is given by $\frac{4 \sqrt{6}}{\pi} v_{D C} \cos (\theta) \angle \phi$.

Given the required voltage magnitude and angle (as ob-

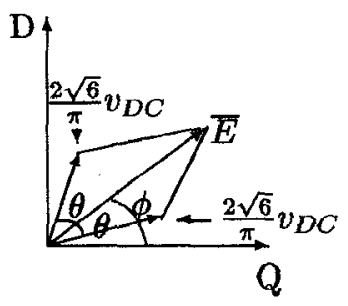

Figure 7: Magnitude control

tained from the controller), the angle $\theta$ can be calculated.

\subsection{Transient Simulation}

Digital Simulation has been carried out using the dynamic system simulation package SIMULINK [7]. The action of the converters is represented by relating the $\mathrm{AC}$ and $\mathrm{DC}$ side quantities using switching functions. The assumption for this study is that the switches are ideal.

Mathematically the relations can be written as (refer Fig. 8)

$$
\begin{array}{r}
v_{a}=S_{a} v_{D C} \\
v_{b}=S_{b} v_{D C} \\
v_{c}=S_{c} v_{D C} \\
i_{D C}=i_{a} S_{a}+i_{b} S_{b}+i_{c} S_{C}
\end{array}
$$

$S_{a}, S_{b}, S_{c}$ are switching functions and are obtained from

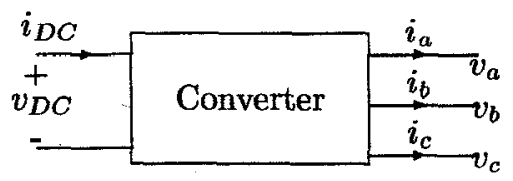

Figure 8: Representation of converter

the firing angle controller. 


\subsection{Simulation Results}

Consider the system shown in Fig. 9.

System data is given in the appendix. For the results

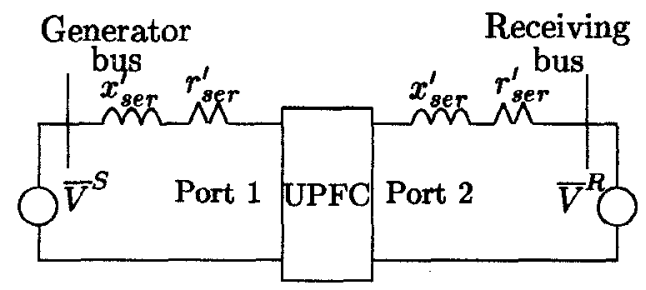

Figure 9: System under Study

$\mathbf{a}, \mathbf{b}$ and $\mathbf{c}$ which follow, we represent the generator by $\mathbf{a}$ voltage source $\overline{V^{S}}$. The generator is modelled in detail for the evaluation of performance of the modulation controller which is presented in $\mathbf{d}$.

(a)StEady State Response

In Figs. 10 we show the steady state waveforms for two operating points:

1. $V^{S}=0.975 \angle 30, P_{R E F}=4.5, V_{R E F}^{u 1}=V_{R E F}^{u 2}=0.975$

To allow for a power flow of which is higher than that with an uncompensated line, the UPFC injects a "capacitive" voltage in series with the line. Also, to maintain the voltage at port 1 , the shunt branch injects reactive power (positive reactive current).

2. $V^{S}=1.0 \angle 30, P_{\text {ref }}=2.5, V_{R E F}^{u 1}=V_{R E F}^{u 2}=0.975$

Here the UPFC injects an "inductive" voltage in order to maintain a power flow of $2.5 \mathrm{pu}$.

(b)RESPONSE FOR A PULSE DISTURBANCE IN THE POWER REFERENCE

The performance of the UPFC for a pulse in the power reference ( 4.5 to 2.5 to $4.5 \mathrm{pu}$ ) is shown in (Fig. 11). The UPFC can respond rapidly (within one cycle) to the changes in power reference. At the same time it maintains its port voltages constant. While reactive voltage is changed in order to change the power flow, the real voltage injection and the shunt reactive current maintain the port voltage magnitudes constant.

In Fig. 11, we have also evaluated the instantaneous magnitude of voltage at ports 1 and 2 as defined in the D-Q variables: $\sqrt{\left(v_{D}^{u 1}\right)^{2}+\left(v_{Q}^{u 1}\right)^{2}}$ and $\sqrt{\left(v_{D}^{u 2}\right)^{2}+\left(v_{Q}^{u 2}\right)^{2}}$ respectively; reactive voltage is evaluated using Eq.5 .

(c)RESPONSE FOR A PULSE DISTURBANCE IN SENDING END VOLTAGE

The capability of the UPFC to regulate power and the voltage at its 2 ports is evaluated for a pulse disturbance in the sending end voltage magnitude ( 1.05 to 0.975 to $1.05 \mathrm{pu}$ ). Fig.12 shows the response of the UPFC. The shunt reactive current (evaluated using Eq. 8) is the quantity which is mainly affected and ensures that bus voltage are regulated.

(d)RESPONSE FOR A PULSE DISTURBANCE IN GENERATOR MECHANICAL TORQUE

We have carried out a simulation study with generator dy-
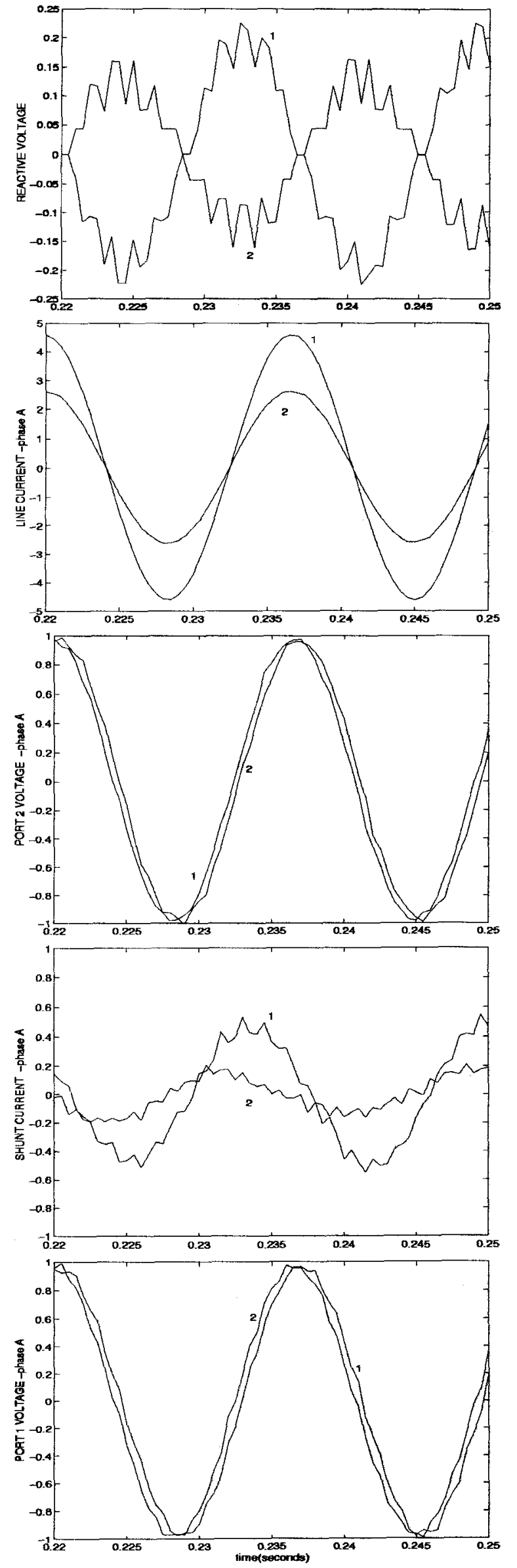

Figure 10: Steady state Waveforms 

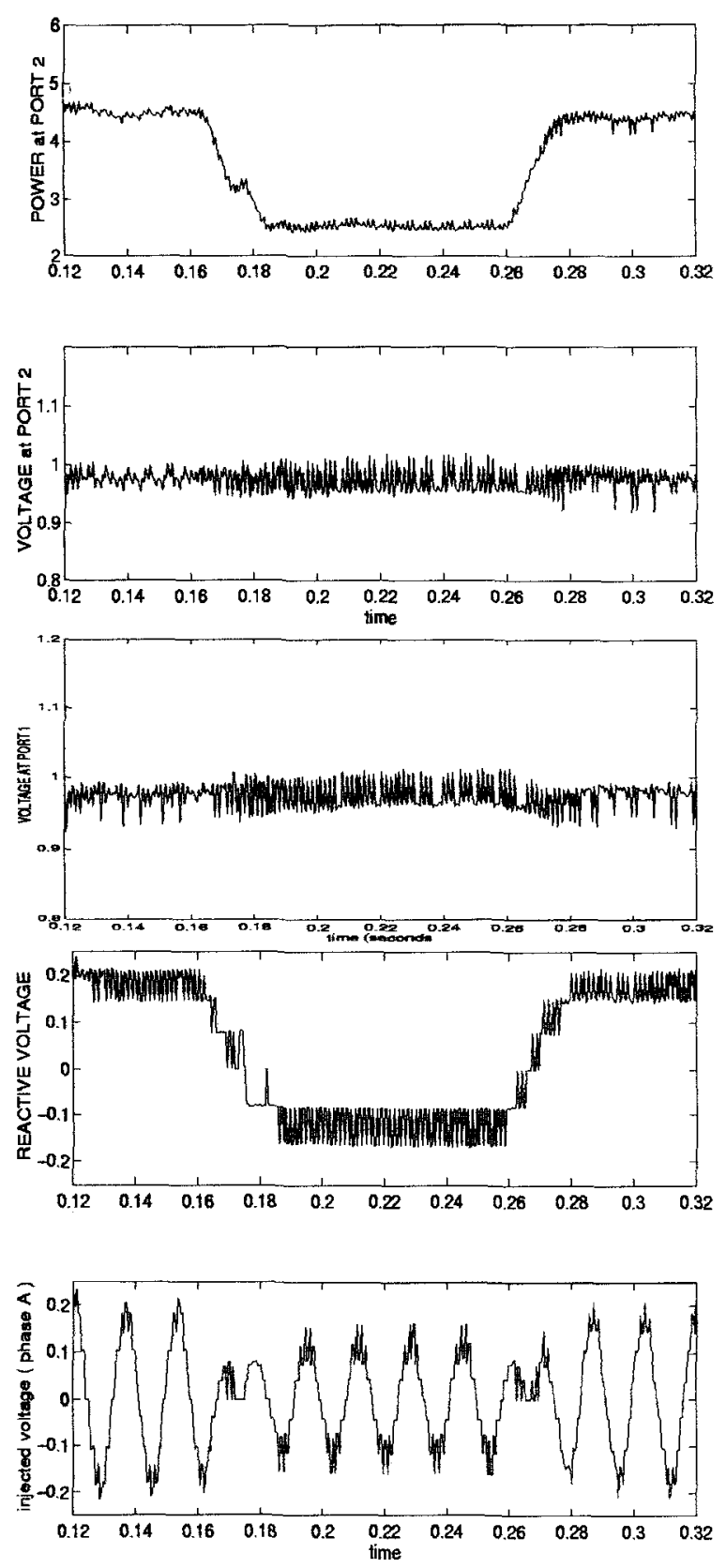

Figure 11: Response to pulse change in $P_{R E F}$

namics included. Besides the dynamics of the rotor inertia ("Swing Equation"), the flux decay equation for the field winding and a damper winding on the quadrature axis is also considered. While Automatic Generation Control dynamics is not considered because it is relatively slow acting, a fast acting Automatic Voltage regulator is considered. Transient saliency is neglected.

To illustrate the effectiveness of the UPFC we consider a pulse disturbance of magnitude 1 p.u. in mechanical input torque. The generator rotor angle tends to have very large deviations and is poorly damped without the UPFC (uncompensated line). On the other hand, the UPFC modulation controller ensures excellent damping as well as restricts rotor angle deviation. It is seen that injected reactive voltage is the major component of the corrrective action taken by UPFC.
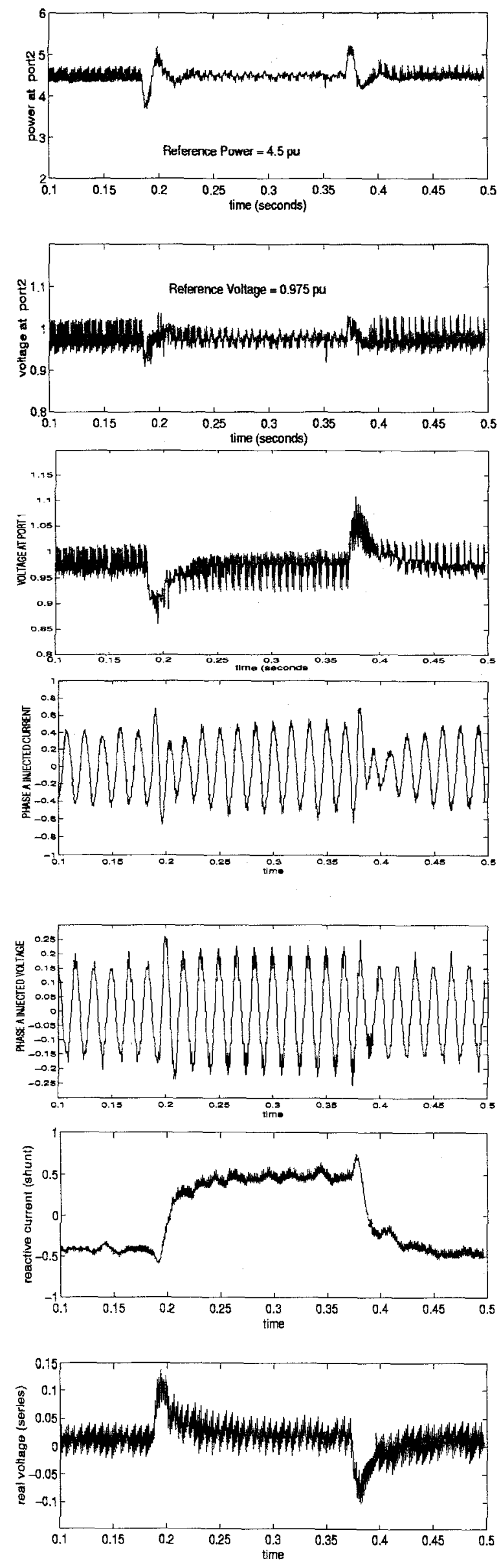

Figure 12: Response to pulse change in sending end voltage 

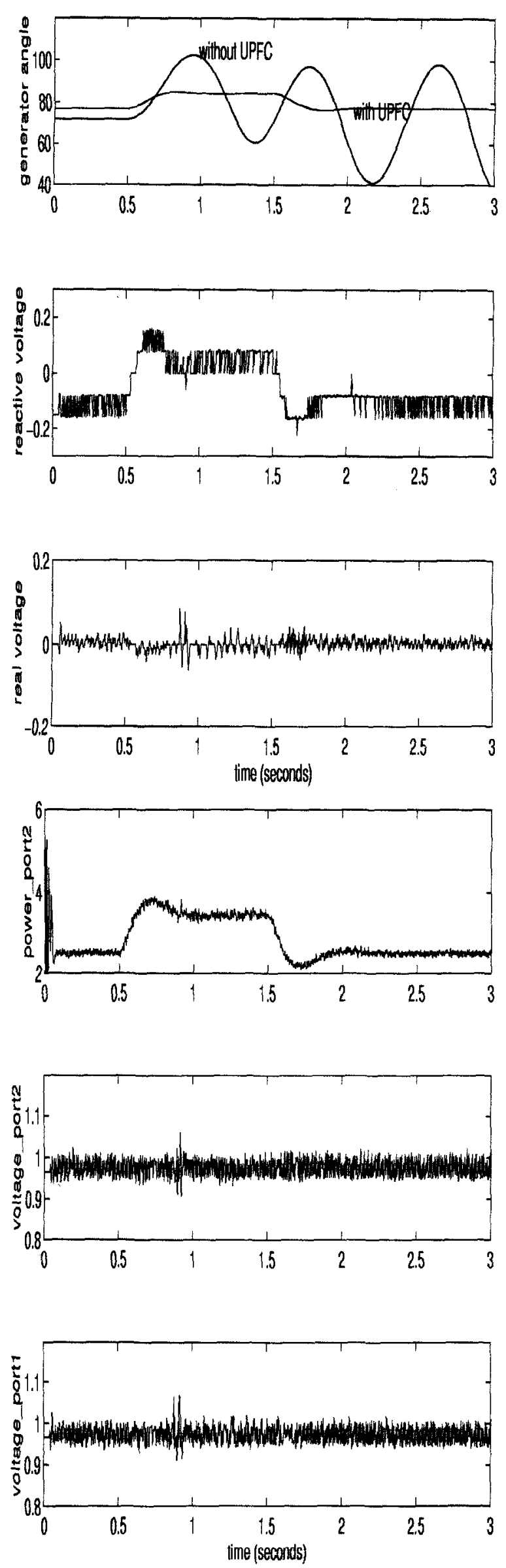

Figure 13: Response for pulse disturbance in input torque

\section{Conclusions}

The performance of the control scheme which employs reactive voltage to control real power flow and real voltage to indirectly control reactive power flow has been investigated using transient simulation. The power reference of the power flow controller is modulated to limit magnitude and improve damping of power swings in the transmission line. The case study conducted indicates that the control scheme is effective in achieving these objectives.

\section{Acknowledgement}

The financial support received from the Dept. of Science and Technology, Govt. of India under the project titled "Flexible AC Transmission Systems (FACTS) controllers" is gratefully acknowledeged.

\section{References}

1 L.Gyugyi et al, "The Unified Power Flow Controller : A new Approach to Power Transmission Control", IEEE Trans. on Power Delivery, Vol. 10, No.2 April 1995, pp. 1085-1097.

2 L. Gyugyi, "Unified power flow concept for flexible AC transmission systems", IEE Proc-C, July 1992, pp.323331.

3 K.R.Padiyar and A.M. Kulkarni, "Development and Evaluation of Controls for Unified Power Flow Controller", Ninth National Power Systems Conference, 1996 , IIT Kanpur, India.

4 K.R. Padiyar and K. Uma Rao, "A Control Scheme for Unified Power Flow Controller to Improve Stability of Power Systems", Ninth National Power Systems Conference, 1996, IIT Kanpur, India.

5 C.Schauder and H. Mehta, "Vector Analysis and Control of Advanced Static Var Compensators", IEE Proc.-C, Vol. 140, No.4, July 1993., pp.299-306.

6 Loren H. Walker, "10-MW GTO Converter for Battery Peaking Service", IEEE Trans, on Industry Applications, Vol. 26, No. 1, Jan/Feb 1990, pp.63-72.

7 SIMULINK Users Guide, The Math Works Inc., 1993.

\section{Appendix}

Nominal system data (on UPFC base which is taken to be $\frac{1}{3}^{\text {rd }}$ of the transmission line base) is given below.

$r_{\text {ser }}^{\prime}=0.0075, x_{\text {ser }}^{\prime}=0.075$,

$x_{s h}=0.15, r_{s h}=0.01$ (shunt transformer reactance and resistance repectively), frequency $=60 \mathrm{~Hz}$.

$b_{\text {cap }}=2.0, g_{\text {cap }}=0.02$ (susceptance and conductance of capacitor repectively)

$\overline{V^{S}}=1.0 \angle 30$ (generator terminal voltage), $\overline{V^{R}}=1.0 \angle 0$ (infinite bus voltage) 